\title{
Calculation of high speed water column pressure on the inner barrel in the gun water-projectile test based on CFD
}

\author{
Zefeng Zhang ${ }^{\mathrm{a}}$, Jianping $\mathrm{Fu}^{\mathrm{b}}$, and Yingxiu $\mathrm{Cao}^{\mathrm{c}}$ \\ College of Mechanical Engineering, Shijiazhuang 050003, China; \\ a972653865@qq.com, ${ }^{b} 2101370148 @ q q . c o m$, mingxiu0910@126.com
}

Keywords: water-projectile test, high speed water column, CFD, two dimensional simulation

\begin{abstract}
It is hard to get the pressure of high speed water column on the inner barrel through the gun water-projectile test. In order to optimize the design, the calculation model of the pressure in the body tube is established. Two dimensional numerical simulation was carried out for gun water-projectile test's high speed water column in the body tube through using Fluent software, the distribution of turbulence was described by using the $k-\varepsilon$ model and the gas-liquid interface of the inside body tube was tracked through two-dimensional VOF model. It was got that simulation of the evolution of the gas - liquid interface image, as well as the pressure distribution at different time in the tube. From the simulation results, it can be seen that the high speed water column will have great pressure on the pipe wall, and the results will be of some guiding significance for the improvement of the gun water-projectile test.
\end{abstract}

\section{Introduction}

During the process of water-projectile test, high temperature and high pressure gas generated by the combustion derived the water column for high-speed movement, it also have similar recoil and counter recoil movement, but compared with the firing, the interior ballistic occurred great changes $^{[1]}$. If the water-projectile test plan is not reasonable ${ }^{[2]}$, such as excessive water quality, it will cause a new plastic deformation of the gun barrel and lead to the bulging phenomenon ${ }^{[3]}$.This phenomenon will cause unnecessary gun water-projectile test safety accident. At present, it was little to carry out the water-projectile test's experimental and theoretical study because of testing personnel by the constraints of technical force. They still explore the safety of water-projectile test through the angle of the practice and experiment. This method is simple and practical, but blindness, prone to bulging phenomenon. The phenomenon of gun barrel bulging in the water-projectile test can only be used as a qualitative analysis, which can not be quantitatively described in theory. In order to avoid the body tube bulge bore and other damage, it is necessary to study the interaction mechanism between the high speed water column and the pipe body.

High speed water column motion in the water-projectile test is a high speed and high pressure gas-liquid two-phase turbulent flow in a large size and short circular tube. Due to the high cost of experiment and simulation, the related research is less. Montanariet Federico and other ${ }^{[4]}$ used the fluid dynamics software Fluent to simulate the water projectile launch and flight process of water cannon. The initial boundary conditions were provided using the results of the calculation of the interior ballistic software for the analysis of the exterior trajectory, and the two phase flow model is used to analyze the complex mechanism of the launch process and the fragmentation of the water projectile during the flight. This paper proves the feasibility of Fluent software to change the shape of water mist and the impact of water on the target. V.Nguyen Anh and other ${ }^{[5]}$ used Fluent software to simulate the gas nozzle impact surface and VOF and $k-\varepsilon$ equation to close the N-S equation, the 3 different gas-liquid interface shapes are obtained at different inlet velocities. CFD software was used to simulate the high speed water column in the tube, which can reduce the cost and make pipe flow field be visual. The two-dimensional numerical simulation of the high speed water column was processed by the CFD software package Fluent software, and it used the VOF model for tracking the gas-liquid interface shape. Finally, the size of tube wall pressure caused by high speed water column was calculated. This paper promotes the further research on the mechanism of 
the interaction between the high speed water column and the body tube.

\section{Principle of water-projectile test}

The principle of water-projectile test is shown in Figure 1. When it launched, the breechblock firing pin impacts cannon primer, and primer can be reliable ignition. The combustion produced the gas with high temperature and high pressure, which push forward the water-projectile (cork and water) in bore high-speed movement until flies away from the gun muzzle. Due to the characteristics of the incompressible water, the water column of high speed motion in the bore can generate a lot of force to the body tube. The calculation of the force is the focus of this paper.

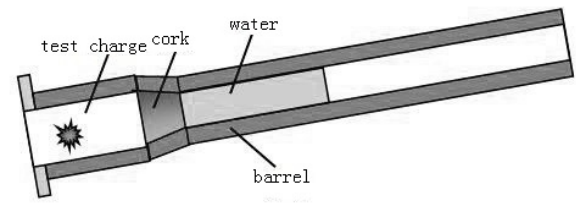

Fig. 1 principle of water-projectile test

\section{Pressure calculation model of high speed water column on the inner wall of the pipe}

\subsection{Basic assumptions}

Under the action of high pressure gas, the water column quickly got acceleration, and the action time was short and the interaction was complicated. In order to be simple for modeling and calculation, it make the following assumptions:

(1) Cork structure was ignored, and pressure distribution behind the water column is uniform and equal in size.

(2) The water column is cylindrical, and the mixing between the gas and the liquid is not considered.

(3) Because the launch time is very short, the gas expansion is isentropic process.

\subsection{Computational model}

\subsubsection{Governing equation}

In the experiment of the gun water-projectile test, because the water is incompressible and the $\rho$ is constant. The continuity equation of the motion in the high velocity water column can be obtained by the mass conservation law ${ }^{[6]}$.

$$
\frac{\partial u}{\partial x}+\frac{\partial v}{\partial y}=0
$$

In this formula, $x$ and $y$ are the bore axial and radial coordinate, $u$ and $v$ are the axial and radial velocity components.

The momentum conservation equation is a basic law that must be satisfied for any flow system. The fluid element in a high speed water column's momentum of time change rate is equal to the differential effects of various external force sum. The momentum conservation equation of the fluid element axial and radial respectively:

$$
\left\{\begin{array}{l}
\rho\left(\frac{\partial u}{\partial t}+u \frac{\partial u}{\partial x}+v \frac{\partial u}{\partial y}\right)=\rho F_{x}-\frac{\partial p}{\partial x}+\mu\left(\frac{\partial^{2} u}{\partial x^{2}}+\frac{\partial^{2} u}{\partial y^{2}}\right) \\
\rho\left(\frac{\partial v}{\partial t}+u \frac{\partial V}{\partial x}+v \frac{\partial v}{\partial y}\right)=\rho F_{y}-\frac{\partial p}{\partial y}+\mu\left(\frac{\partial^{2} V}{\partial x^{2}}+\frac{\partial^{2} V}{\partial y^{2}}\right)
\end{array}\right.
$$

In this formula, $\rho$ is the element density, $u$ and $v$ are the axial and radial velocity components, $\mathrm{t}$ is time, $\mathrm{x}$ and $\mathrm{y}$ are the bore axial and radial coordinate, $p$ is the center point of the pressure, $\mu$ is dynamic viscosity, $F_{x}$ and $F_{\mathrm{y}}$ are the sun force that acting on the element in the direction of $x$ and $y$.

A turbulent model is needed to describe the turbulent motion of high speed water column in the 
body. In this paper, a standard $k-\varepsilon$ model which can simulate the process of turbulent flow is used to describe the turbulent motion. The model needs to solve the turbulent kinetic energy and its dissipation rate equation. The turbulent kinetic energy transport equation is obtained by exact equation, and the dissipation rate equation is obtained by physical reasoning and mathematical simulation. The turbulent kinetic energy $\boldsymbol{k}$ and dissipation rate $\varepsilon$ equations are described as follows $^{[7]}$ :

$$
\left\{\begin{array}{l}
\rho \frac{d k}{d t}=\frac{\partial}{\partial x_{i}}\left[\left(\mu+\frac{\mu_{t}}{\sigma_{k}}\right) \frac{\partial k}{\partial x_{i}}\right]+G_{k}+G_{b}-\rho \varepsilon-Y_{M} \\
\rho \frac{d \varepsilon}{d t}=\frac{\partial}{\partial x_{i}}\left[\left(\mu+\frac{\mu_{t}}{\sigma_{\varepsilon}}\right) \frac{\partial \varepsilon}{\partial x_{i}}\right]+C_{1 \varepsilon} \frac{\varepsilon}{k}\left(G_{k}+C_{3 \varepsilon} G_{b}\right)-C_{2 \varepsilon} \rho \frac{\varepsilon^{2}}{k}
\end{array}\right.
$$

In this formula, $\rho$ is High speed water column density, $k$ is turbulent kinetic energy, $t$ is time, $\mu$ is dynamic viscosity, $\mu_{t}$ is turbulence intensity, $\sigma_{k}, \sigma_{\varepsilon}$ is turbulent Prandtl number, $G_{k}$ is the generation of turbulent kinetic energy due to the mean velocity gradient, $G_{b}$ is turbulent kinetic energy generated due to the influence of buoyancy, $\varepsilon$ is dissipation rate of turbulence, $Y_{M}$ is effect of compressible turbulent pulsation expansion on the rate of dissipation, $\mu_{t}$ is turbulence intensity, $C_{1 \varepsilon}$ 、 $C_{2 \varepsilon}, C_{3 \varepsilon}$ is constant.

\subsubsection{VOF model}

VOF model is a kind of surface tracking method in fixed Euler grid by solving separate momentum equation and processing through the region of the volume fraction of each fluid to track incompatible fluid between the interfaces. By using the model to track the gas-liquid interface, the shape of the gas-liquid interface is obtained.

The interface between the high speed water column and the high pressure gas is accomplished by solving the continuous equation of the volume fraction of the two phases. The gas phase and the liquid phase is calculated based on follows constraints:

$$
\sum_{q=1}^{n} \alpha_{q}=1 \quad(n=2)
$$

In this formula, $\alpha_{q}$ is volume fraction of q phase.

\section{Calculation of inner wall pressure of high speed water column based on CFD}

\subsection{Structure and mesh generation}

In this paper, the gun barrel is calculated as the calculation area, as shown in Figure 2. Due to the rule of the region, mesh is relatively simple. Because the pipe wall region pressure gradient changes dramatically, so close to the pipe wall flow region encrypted by the grid technology. And combining with the computer calculation ability to in this region are properly encrypted which can improve the calculation precision of the region. In the middle part, the uniform linear mesh generation technique is adopted.

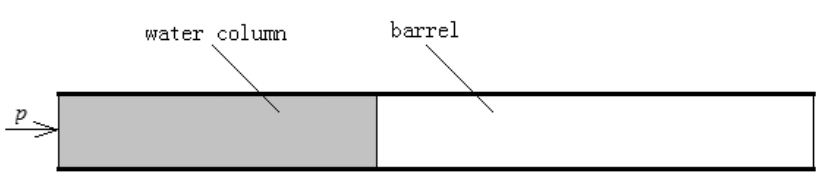

Fig. 2 Calculation area

The grid is divided into 27492 quadrilateral structure grids and 28197 nodes, which are generated by the Fluent pre-processing software Gambit. The computational grid can be divided into structural mesh, unstructured grid and hybrid grid. Calculation of the area using the structure grid, the grid can be conveniently and accurately processing boundary conditions and improve the calculation accuracy, at the same time, selection of the grid can use many implicit algorithms and 
multigrid methods, computational efficiency is also higher. Figure 2 shows a portion of the grid graph.

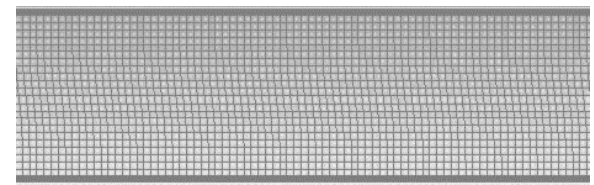

Fig. 3 Local grid graph

\subsection{Boundary conditions and initial conditions}

Taking a test gun as an example, the pressure change in the bore is calculated by the interior ballistic trajectory, as shown in Figure 4. The result was to be initial inlet pressure boundary condition .Water column length is $1.199 \mathrm{~m}$, initial velocity is 0 . The pressure outlet boundary condition is outlet boundary condition and the outlet pressure is atmospheric pressure. The remaining default is wall.

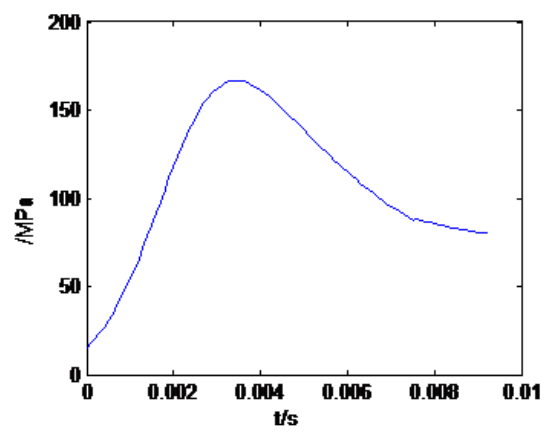

Fig. 4 Pressure change with time in bore

\subsection{Simulation results and analysis}

The numerical simulation of the physical process of the gun in the tube is carried out through the Fluent software. The simulation is completed on the computer (CPU type: Intel core I3 2330M, Graphics chip,GeForce GT 540M NVDIA). The time step is set to $0.01 \mathrm{~ms}$ and the calculation time is $4 \mathrm{~h}$.

4.3.1 VOF tracking of gas-liquid interface

The water column movement can be tracked by the VOF method. Fig. 5 shows the position of water column in the body tube for $0.008 \mathrm{~s}$. And the high speed water column in pipe body moving in a cylindrical remained unchanged. At the same time, the wall has a little friction holding liquid group. The shape of the gas-liquid interface is basically symmetrical distribution, which shows that the effect of inertia force (pressure) is more obvious than the gravity when the water column is moving in the $3.5 \mathrm{~m}$ tube.

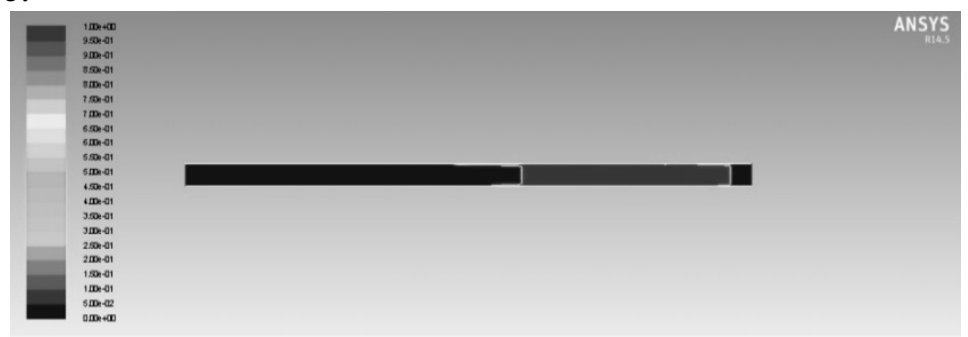

Fig. 5 Gas-liquid interface of $0.008 \mathrm{~s}$

Through the analysis of the data, the position of water column in the pipe at different times is obtained, as shown in figure 6. 


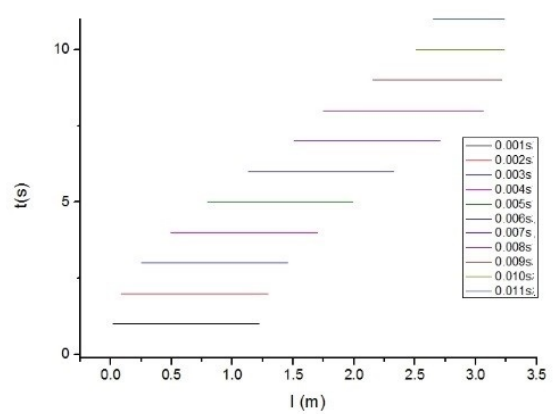

Fig. 6 Position of water column at different times in tube

\subsubsection{Distribution of pressure}

Pressure as the power source of the system promote the water column for high-speed movement. Due to inertia, the water column will have a huge force on the tube wall. Through the simulation calculation, we can get the pressure of a certain point of time and the pressure distribution on the tube at a certain time. Fig. 7 shows distribution of pressure in the body tube for $0.008 \mathrm{~s}$. It can be seen that there is a clear turning point in the color of the position, that is, the location of the gas liquid interface. In the rear of the interface is the high pressure air chamber, and the pressure value is basically the same. In front of the interface is the water column that the pressure gradient is relatively large.

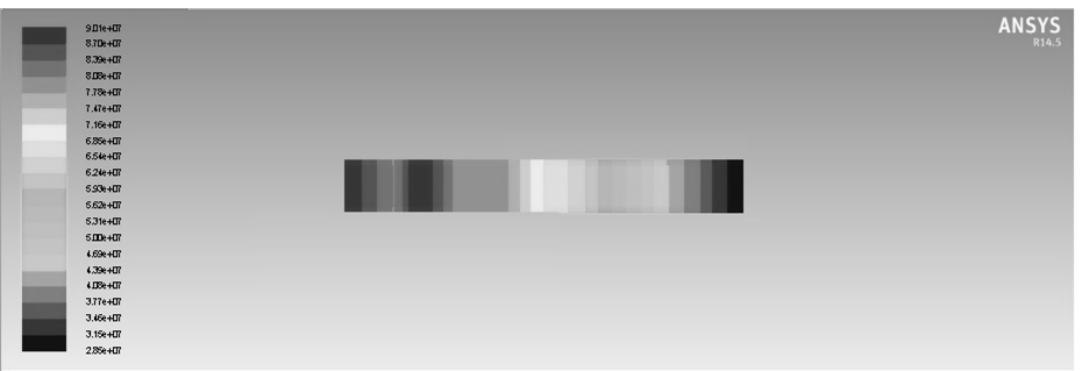

Fig. 7 The distribution of pressure in the body tube during $0.008 \mathrm{~s}$

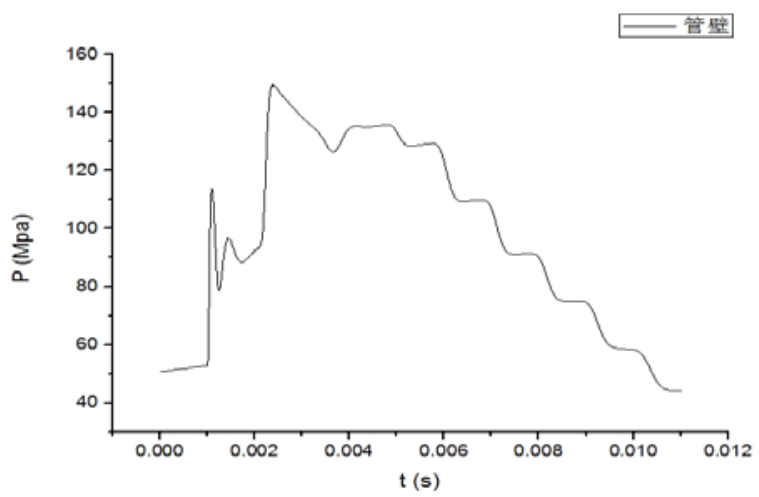

Fig. 8 The distribution of pressure in the body tube during $0.008 \mathrm{~s}$

Figure 8 shows the variation of the wall pressure with time, which is from the gun $2636.5 \mathrm{~mm}$.There are two wave peak, the first peak appeared at the $0.00144 \mathrm{~s}, 96.78 \mathrm{Mpa}$, the second peak appears in 0.00241s 149.46 Mpa. It can be known from figure 6 that the 2 peaks are in the water column. Figure 8 shows the variation of the wall pressure with time, which is from the gun $2636.5 \mathrm{~mm}$.there are two wave peak, the first peak appeared at the $0.00144 \mathrm{~s}, 96.78 \mathrm{Mpa}$, the second peak appears in 0.00241s 149.46 Mpa. It can be known from figure 6 that the 2 peaks are in the water column.

\section{Conclusion and prospect}

In this paper, the Fluent software is used to simulate the high speed water column in the water-projectile test, and some conclusions are obtained. 
(1) The pressure at the rear of the gas liquid interface is almost equal, and the pressure in the front gradually decreases.

(2) The inertia force (pressure) than gravity on the shape of the gas-liquid interface is more obvious

(3) High speed water column will have a great force on the body tube of gun.

Although the simulation results have been some conclusions, but it is still necessary to verify the experimental results with the actual situation. By means of modern test technology, the size of force will be tested to validate weather the simulation calculation model is correct, which is one of the key work in the future.

\section{References}

[1]. FU Jian-ping,LV Shi-le,ZHEN Li-ping, et al. Study on the ballistic missile test of artillery projectile[J]. Journal of Ordnance Engineering College. Mar. 26(2014), p.21-24.

[2]. DIAO Zhong-kai. Discussion on the shooting scheme of artillery projectile[J]. Ordnance repair engineering research.Feb.(2001) p.63-65.

[3]. ZHOU Ming-gao. On the mechanism of the water projectile bulging[J]. Sichuan ARMAMENTARII. Mar.(1982), p.34-36.

[4]. Fedderico M on tanari et al Exploratory CFD Analyses of the Fluid Dynamics of a Water Cannon[C]//AIAA Aerospace Science Meeting and Exhibit,2005

[5]. Anh V N,G eoffrey M E. Computational Fluid Dynamics Modelling of Gas Jets Impinging Onto Liquid Pools[J].Applied Mathematical Modelling 2006(30):1472-1484.

[6]. Yang Guo-lai, Zhou Wen-hui, Liu Fei. Flow field simulation of high pressure water jet nozzle based on FLUENT[J]. Journal of Lanzhou University of Technology. Mar. 34(2008), p.49-52.

[7]. Yu Hua-bing,Kang Shi-ting,Hu Ren-xi. Fluent14.5 flow field analysis[M]. Machinery Industry Press.2014. p.206-207. 